\title{
Literarische Erfolgsbildung - am Beispiel Walter Kempowski
}

\section{GÜNTHER MAHAL}

Den Erfolg eines Autors an der Wirkung auf seine Leser oder an seinem Einfluß auf andere Autoren zu messen, festzustellen also, ob und in welchem Maße ein Autor Zustimmung, Irritation oder Ablehnung, stilistische Distanz, inhaltliche Auseinandersetzung oder Nachahmung hervorruft - eine Erfolgskontrolle in diesem Sinne ist sehr schwer und allenfalls durch genaue Auswertung individueller Rezeptionsaussagen durchführbar. Voraussetzung dafür ist außerdem ein zeitlicher Abstand von' ausreichender Länge - ausreichend dafür, um nicht nur ephemere Wirkungsreflexe und kurzlebige Erfolgsechos einzufangen, sondern um breit belegen zu können, seit wann, warum, in welcher Weise und Stärke und mit welcher Kontinuität das Werk eines Autors die Leser und Kollegen beeinflußt und möglicherweise zur Realisierung anempfohlener Denkbewegungen oder Handlungsappelle angestoßen hat.

Für einen Autor wie Walter Kempowski, der vor erst elf Jahren mit seinem Bautzener Zuchthausbericht Im Block debütierte, wäre die angedeutete Methode der Erfolgsmessung ganz gewiß verfrüht und auch - von der besonderen Art seines Schreibens her - verfehlt und müßig; über die Gründe dieser Behauptung, über die durch das Romanwerk Kempowskis selbst planvoll verhinderte Leserreaktion im oben beschriebenen Sinn wird zu sprechen sein.

Es geht im folgenden nicht um einen Versuch empirischer Leserforschung, der durch Interviews, Fragebogen-Aktionen oder ähnliche Instrumentarien zu dem Ziel zu kommen hoffte, Repräsentativität der Rezipienten-Aussagen ebenso $\mathrm{zu}$ erreichen wie ein nach Lesertypen strukturiertes Modell differenziert aufgenommener und beantworteter Wirkungssignale. - Auch ein solches Verfahren, so soll sich zeigen, wäre im Falle Kempowskis unangemessen. Was sich am breit gestreuten Erfolg dieses Autors erkennen läßt und was vornehmlich vom Autor und seinen Texten her, nicht so sehr von der Rezipientenseite aus untersucht werden soll, stellt einen Extremfall literarischer Erfolgsbildung dar. Die an diesem Extremfall gewonnenen Ergebnisse für andere Autoren fruchtbar zu machen, wäre Gegenstand weiterer Untersuchungen.

Was sich im Blick auf das Stichwort 'Erfolg' für Kempowski empirisch, mit statistischer Absicherung, sagen läßt, das sind quantitative und unbestreitbare Befunde, Verkaufszahlen nämlich, die ihn selbst und seine beiden Verlage - 
lange Zeit Hanser, seit kurzem Knaus - demnächst zu Auflagen-Millionären machen werden. Der eben fünfzigjährige Kempowski, 'Landlehrer' mit halbem Deputat im niedersächsischen Nartum, hat seit 1969 eine erstaunliche Fülle schriftstellerischer und publizistischer Arbeiten vorgelegt, und er ist mit gutem Recht als ein disziplinierter und fleißiger Autor gerühmt worden, als Schreibtischhandwerker, der seine immensen Zettelkästen bereitwillig herzeigt und dem Worte wie Inspiration oder Dichtertum zumindest coram publico nicht von den Lippen gehen. Kempowski veröffentlichte mehrere Hörspiele, einen Bildband über seine umfangreiche Zinnsoldatensammlung, drei Umfragebücher, die mit seinen Romanen im "Verbundsystem" 2 zu sehen sind; er interviewte (sehr loyal übrigens, um das Mindeste zu sagen) Helmut Kohl und Albert Speer; er ließ eine bibliophil ausgestattete Lehrer-Erzählung (Unser Herr Böckelmann) erscheinen, unverkennbar ein nur leicht verfremdetes Selbst-Porträt; er schrieb Kinderbücher und plant eine Fibel, einen Kriminalroman und eine Indianergeschichte. ${ }^{3}$ - Am meisten Beachtung aber fanden und finden die vier Romane, die zusammen mit zwei noch folgenden Teilen einen Zyklus bilden sollen, der die Schicksale seiner eigenen Familie Kempowski beschreibt, angefangen von der kaiserlich-wilhelminischen Epoche bis zur bundesrepublikanischen Gegenwart.

1971 erschien zunächst Tadellöser \& Wolff, im Untertitel Ein bürgerlicher Roman, der die Nazizeit und den zweiten Weltkrieg umfaßt; 1972 folgte Uns geht's ja noch gold, der Roman einer Familie mit dem erneut vorangestellten kokettierenden Motto "Alles frei erfunden!" - dieser Roman behandelt die Nachkriegszeit 1945 bis 1948. Die folgenden acht Jahre, die Zeit bis 1956, sind in Ein Kapitel für sich dargestellt, einer Umarbeitung von Kempowskis literarischem Erstling Im Block - das Buch erschien 1975. Schließlich erschien 1978 der vorläufig letzte Teil von Kempowskis Familienchronik, der nach den bisherigen Planungen des Autors den Beginn seines Romanzyklus bilden soll: Aus großer Zeit - er umfaßt die wilhelminische Ära, die Zeit von Kempowskis Großeltern, und er reicht bis zum Ende des ersten Weltkriegs. Die jetzt noch ausstehenden Teile der am Ende vier Generationen umspannenden 'Familiensaga', nämlich die Zeiträume von 1918 bis 1933 und von 1956 bis zur Gegenwart, sollen in Bälde vorliegen. In einem sogenannten Null-Buch will Kempowski einigen kritischen Einwänden gegen die Romanfolge entgegentreten und "den Versuch wagen, die Gründe für den Militarismus meines Vaters oder die wilhelminische Autoritätsgläubigkeit meiner Mutter zu finden, um zu erfahren, wie es zu der Katastrophe [gemeint ist die deutsche Katastrophe] kommen konnte." 4 Als Gesamttitel dieser deutsch-bürgerlichen Rougon-Macquart, dieser allen Widrigkeiten trotzenden Comédie humaine, ist ein Name der griechischen Mythologie vorgesehen, den Camus in anderem Kontext verwendete: Sisyphus.

Einer von Kempowskis Romanen, Tadellöser \& Wolff, ist 1975 einem vielmillionenfachen Publikum in einer Fernseh-Verfilmung vorgeführt worden; nach der Ausstrahlung des Films, bei dem Kempowski als Berater fungiert hatte, schnellten die Verkaufszahlen seiner Bücher beachtlich in die Höhe; 
die Reaktionen auf diesen Film über das Leben einer deutschen Familie während der Naziherrschaft hat Walter Jens auf die Formel gebracht: "Von rechts bis links ein einziger Begeisterungssturm: Jawohl, so ist es gewesen!" Die scheinbare Paradoxie dieses bedenklich ausgewogenen "Jubelschreis"5 läßt sich freilich - das wird zu zeigen sein - leicht auflösen.

Angemerkt sei noch, daß Kempowskis Romane in den Leihbüchereien - jenen meist zu wenig beachteten Indikatoren der breiten Lesergunst - sogenannte 'Dauerläufer' darstellen, in der Regel also nur aufgrund wochenlanger Vorbestellungen zu bekommen sind. Erwähnenswert ist auch, daß die Wochenzeitung Die Zeit am 8. April 1977 dem Kempowski-Boom auf nicht alltägliche Weise Tribut zollte: auf zwei Ganzseiten wurde hier dokumentiert, wie es zur Ablehnung von Kempowskis erstem Manuskript durch den Rowohlt-Verlag gekommen war - eine Hommage, die nicht ohne Peinlichkeiten blieb, Peinlichkeiten, die sich keineswegs auf Verlag und Gastlektoren beschränkten. ${ }^{6}$ Kempowski, der als 'Liberaler aus Überzeugung' gelten möchte, ${ }^{7}$ der längst schon eine "Figur des Literaturbetriebs, der Schullektüre und der gedankenvollen Kaffeekränzchen"8 geworden ist, gehört dem PEN an, ohne die Veranstaltungen des Clubs zu besuchen. Er stellt sich gern Interviews und reist bereitwillig zu Lesungen und Tagungen, und er hat - auch dies zweifellos ein Indikator seines Erfolges - bisher vier Literaturpreise erhalten. ${ }^{9}$

Als Summe solcher Einzelheiten ergibt sich das Bild eines mittlerweile sehr bekannten, ungewöhnlich produzierfreudigen und auch von merkantilem Erfolg belohnten Autors, der längst seine 'Gemeinde' hat und dessen nächster zum familienchronikalischen Zyklus gehöriger Roman sicherlich mit einer noch höheren Startauflage herauskommen wird als der zuletzt erschienene Kaiserzeit und Weltkriegs-Teil Aus großer Zeit; dieser Roman wurde mit 50000 Exemplaren aufgelegt. - Diese Zahlengrößen werden in der deutschsprachigen Gegenwartsliteratur nur von ganz wenigen Autoren erreicht - von Grass, Lenz, Böll, Walser etwa; vergleichbare Größenordnungen erreichen ansonsten nur Gymnasialklassiker oder Trivialliteraturproduzenten. (Indessen würde diese Zusammenstellung von Schiller und Simmel oder Kleist und Konsalik als effekthascherisches Bonmot oder als provokative Unnötigkeit mißverstanden: der Erfolg Walter Kempowskis legt die Frage nahe, ob er als ein immergültige Antworten bereithaltender Klassiker rezipiert wird oder ob er mit Schreibstrategien trivialliterarischer Art ein immer größeres Publikum zu gewinnen weiß - oder ob er es gar versteht, diesen beiden sehr disparaten Aspekten in gleicher Weise Rechnung zu tragen. Er selbst, so äußerte er in einem Interview mit Jost Nolte, "möchte wissen, ob ich nun zu Simmel gehöre oder zu Grass und den andern. Natürlich würde ich lieber zu Grass und seiner Klasse gehören."10)

Schon 1972 hat Peter Wapnewski in einer sehr einlässigen Rezension des Romans Uns geht's ja noch gold postuliert: "angesichts des beachtlichen und beachteten Erfolgs seiner Bücher ist es Aufgabe der Literaturkritik, nach den Voraussetzungen dieses Erfolges zu fragen, nach den Mitteln also eines Erzählens 
von solcher Wirkung (wobei dahinsteht, ob und wieweit überhaupt poetologisches Kalkül beteiligt ist)." 11 - Wapnewskis Monitum blieb innerhalb der Literaturwissenschaft ungehört. Die Erwähnungen Kempowskis in Autorenlexika oder in Überblicksdarstellungen der Gegenwartsliteratur sind zwar häufig, beschränken sich aber meist auf die Aufzählung seiner Werke und auf Kurzcharakteristiken seines unverwechselbaren Stils (übelgesonnene Kritiker, deren Kempowski nicht viele hat, nennen es Masche), also der kurzen Abschnitte von sieben oder zehn oder vierzehn Zeilen, des meist so genannten Pointillismus, der reichen Verwendung von Redensartlichkeiten, von Kalauern, von Schlagerfetzen oder Reklamespots, der minuziösen Wiedergabe von 'Realität', der exakten Recherchen, der Stimmigkeit aller Details u.s.f.

Eine größere Arbeit über Kempowski liegt nicht vor; zwei oder drei kleine Aufsätze stellen eher paraphrasierende Miszellen dar als Versuche, das Oeuvre Kempowskis für sich allein oder auch im Vergleich mit anderen Werken zu interpretieren - es also neben die schon erwähnten Romanzyklen Balzacs oder Zolas oder neben Brochs Schlafwandler zu stellen oder neben die Dramenfolge Carl Sternheims Aus dem bürgerlichen Heldenleben. Der von Kempowski wiederholt und nur wenig verschämt behaupteten Verwandtschaft der Rostocker 'Familiensaga' mit Thomas Manns Lübecker Buddenbrooks ist man bisher noch nicht kritisch nachgegangen. ${ }^{12}$ Und nur in Rezensionen - ich hatte etwa fünf Dutzend vorliegen - erscheinen gelegentlich, wenn auch nur in Andeutungen, Hinweise darauf, was im erhellenden Nebeneinander Licht werfen könnte auf Kempowskis Eigenart: Hinweise auf Jean Pauls vergnügtes Schulmeisterlein auf Solschenizyns Ein Tag im Leben des Iwan Denissowitsch, auf Grass' Oskar Matzerath, auf die Kahlschlagliteratur der frühen 47er, auf Gustav Freytags Ahnen, auf Fontanes Romanwerk, 13 auf Uwe Johnsons Jahrestage, auf die Lebensläufe des gleichfalls mit der 'Pinzette' arbeitenden Alexander Kluge. ${ }^{14}$. Der Hinweis auf ein in der Fontane-Bismarck-Epoche äußerst beliebtes und in Fortsetzungen erschienenes Romanwerk bürgerlich-familiären Zuschnitts und ellbogenhaft-resoluten Privat-Darwinismus fehlte bisher, und dies sicherlich zu Unrecht: der Hinweis auf Julius Stindes Buchholz-Zyklus. Hier läge, so meine ich, eine überaus fruchtbare und ergiebige Möglichkeit des Vergleichs - nicht im Stilistisch-Formalen, wohl aber in der Atmosphäre beider Roman-Reihen, im Vergnüglichen, im Unterhaltlichen, 15 in der kurzen Lupen-Perspektive (oder der "Kniebeugen-Perspektive"16 oder der "in eventu-Perspektive"17), in der Verengtheit und Beschränktheit des Blicks, im Hängen am Detail, in der Grundstimmung eines pläsierlich-angestrengten Sichdurchwurstelns, das alle öffentlichen Großwetterlagen nimmt, wie sie eben kommen. Doch bis sie in die guten Stuben der Buchholzens oder der Kempowskis vordringen, bleibt von ihnen meist nichts übrig als der Anlaß zu Schnacks und zu einer Form von Ironie, die im Grunde diesen Namen gar nicht mehr verdient, weil sich die Kommentare der Romanfiguren dem von außen Kommenden gegenüber nicht abständig zeigen, sondern allem von fernher Eindringenden lediglich Aufkleber 
integrierender Heimeligkeit verpassen.

"Der Bucherfolg", so hat es Siegfried Kracauer definiert, "ist das Zeichen eines geglückten soziologischen Experiments, der Beweis dafür, daß wieder einmal eine Mischung von Elementen gelungen ist, die dem Geschmack der anonymen Lesermassen entspricht." 18 Jürgen Peters hat in seinem Kleinen Versuch über den großen Erfolg Kracauers Feststellung um eine weitere wichtige Komponente ergänzt: "Erfolge haben ihre eigene irrationale Dynamik. Sie wird ermöglicht durch die Identifikations-Disposition der potentiellen Käufer."19 - Beiden Momenten, dem geschmackssoziologischen wie dem unbewußt identifikatorischen, die als einander komplementär betrachtet werden können, beiden Momenten hat die zünftige Literaturkritik im Falle Kempowskis merkwürdigerweise kaum Rechnung getragen. Wenn auch zusätzlich zu diesen beiden Momenten noch eine ganze Reihe weiterer Aspekte hinzutreten müßte, um den Erfolg Walter Kempowskis hinrèichend erklären zu können - die Beachtung der von Kracauer und Peters gegebenen Hinweise, die sich auch andernorts und schon sehr viel früher finden lassen (etwa in Schillers Bürger-Rezension mit ihren aktuell gebliebenen Überlegungen zur Popularität eines Schriftstellers), die Beherzigung also sehr fundamentaler Einsichten in die Voraussetzungen und in das Funktionieren von Erfolg wäre einer emsigen Rezensententätigkeit gut angestanden, die den Erfolg Kempowskis festzustellen zwar nicht müde wurde, die sich aber vor seiner Begründung damit drückte, daß immer wieder dieselben formalen, stilistischen, strukturellen Beobach tungen repetiert wurden.

$\mathrm{Da}$ Kempowski in seinen Romanen eine wissenschaftlich-mikroskopische, zuweilen 'kriminalistisch' genannte, botanisierend sorgfältige, 20 akribisch-archäologisch sammelnde, in jeder Einzelheit verläßliche, kurzum eine authentische und realistische oder auch veristische Art historisch genau situierter Wirklichkeitswiedergabe leisten würde - das wurde oft betont und das schien eine unbestreitbare Charakterisierung; eine Erklärung des Erfolges war es nicht. Zudem darf, gerade vom erwiesenen Erfolg Kempowskis her, in Zweifel gezogen werden, ob ein bloßer Verismus tatsächlich auf ein derart breites Leserecho gestoßen wäre. Anders und genauer: es darf vermutet werden, daß die auch für Kempowskis Romane 'von rechts bis links' erkennbare 'Begeisterung' eher daher rührt, daß die nur im einzelnen Detail tatsächlich veristisch arrangierten Erzähl-"Häppchen"21 keinesfalls eine Wirklichkeitswiedergabe leisten, die in sich konsistent, homogen und dicht, auf sich selbst beschränkt und nach außen hin, auch zum Leser hin abgeschottet wäre. Vielmehr liegen hier durch viele einzelne Realitätspartikel konstituierte Rasterbilder vor, die sich dem Leser gegenüber gleichsam porös erweisen und die mithin seine je persönliche Einlassung, interpretierende Besetzung oder anverwandelnde Erinnerung nicht nur gestatten, sondern geradezu fordern.

Das "Jawohl, so ist es gewesen!", das bestätigend-beglückte Sich-Wiedererkennen vieler Kempowski-Leser, das auch in manchen Rezensionen bewundernd artikulierte "Jetzt erinnere ich mich wieder"22 - oft übrigens artikuliert mit den 
Obertönen aufgekratzter Klassentreffen-Nostalgie23 - die Lektüre-Reaktion also, als erlebe man in Kempowskis Romanen die eigene Vergangenheit authentisch oder gar objektiv wieder, spricht gerade gegen die oft behauptete Authentizität und Objektivität dieser Romane. - Denn dem Autor geht es keineswegs um einen unverwechselbaren Fall, um ein über Generationen hinweg verfolgtes Privat-Schicksal einer zufälligen Familie. Ganz im Gegenteil: ihm geht es - ohne $\mathrm{da} ß$ dies je expliziert würde - um eine Chronik der Kempowskis, die von vornherein auf Typik und Repräsentativität ausgerichtet ist; ihm geht es um ein mit tausend geschichtlichen Realien gespicktes und mit noch mehr assoziationsfördernden Leerstellen versetztes Medium, dessen nur vordergründige Zufälligkeit auf eine breite und sogar auf eine höchst unterschiedliche Identifikation angelegt ist, 'von rechts bis links'; ihm geht es um einen Fall, der verwechselt werden will - der nämlich vom Leser verwechselt werden soll mit seinem eigenen, mit seinem tatsächlich eigenen oder aber mit dem, was verklärende Distanz oder aktive Verdrängung aus ihm gemacht haben.

In Kempowskis Romanen bleibt es in der Tat "jedem Leser überlassen, was er aus seiner Familie einbringen kann"; hier gibt es "auch für Verdränger todsicher ein Wiedersehen mit der Vergangenheit".24 'Todsicher' - diese Vokabel ist gewiß wenig glücklich, könnte man ihr zufolge doch mutmaßen, in Kempowskis Zyklus fände eine grimmige Abrechnung statt, mit deutscher Geschichte, mit deutscher Bürgerlichkeit insbesondere. So ist es zwar von einigen Rezensenten gesehen worden; doch in derlei Befunden entbirgt sich eher der individuelle Erfahrungs- und Denkhintergrund der Schreiber, als daß eine angemessene Deskription des 'Kempowski-Prinzips'25 stattfände. Walter Kempowski hat - in einem Interview mit Dieter E. Zimmer - die in seinen Romanen häufig bemerkte Autorenzurückhaltung 26 als bewußte Schreibstrategie erklärt und im Blick auf die gewünschte Weise der Aneignung seiner Bücher gesagt: "In meinen Romanen führe ich eine Art Puppentheater vor. Ich lasse die Menschen so agieren, daß der Leser dahin geführt wird, ihr Schicksal als das seine zu erkennen, sich mit ihnen zu identifizieren"; und: der Leser soll "sich selber Gedanken machen".27 Als Ergänzung zu dieser vom Autor intendierten identifikatorischen Anverwandlung und zu der gedanklichen - hinzuzufügen wäre: auch der emotionalen - Supplierung der Romantexte durch die Leser hat Kempowski zur Rolle des Autors bemerkt: "Mit Kommentaren bin ich vorsichtig. Jeder, der kommentiert, macht sich ja schuldig."28

Mag sich dieser letzte Satz beim ersten Hören auch plausibel ausnehmen, vielleicht sogar menschenfreundlich in kommentiersüchtigen und deklarationsversessenen Zeiten, so stellt er für Kempowskis Romane doch eine apologetische Maxime dar, an der sich gleichzeitig die Erfolgsfrage seines Werkes ein Stück weiter klären läßt. Denn der so liberal wirkende Satz vom Schuldigwerden durch Kommentieren erhält auch dann oder gar erst Sinn, wenn man ihn auf die Füße stellt: schuldig macht' sich, wer - 'deutungsabstinent'29 - nicht kommentiert, dann nämlich, wenn eine Thematik, ein Erzählzusammenhang, ein 
zeitgeschichtlicher Kontext, ein Syndrom politischer Verirrung oder eine HochZeit ideologischen Aberwitzes nur in der Oberfläche, nur im Reflex, nur als ein ins Privateste zurückgenommener und längst abgeflachter Ausläufer sichtbar wird. ${ }^{30}$ Kempowskis ganz konsequent durchgehaltene Methode, das Bürgerhaus selbst der Nazizeit fern von allem Grollen und Toben und Morden draußen zu halten, das Fliegen des Zeitpulses lediglich am dafür kaum geeigneten Barometer der guten Stube abzulesen, die Geschäfte der Schergen und das Meucheln der Mächtigen über dem Abhören von Feindsendern mit artfremder Niggermusik zu ignorieren - all diese 'brutale Arglosigkeit'31 einer genau berechnenden 'Aussparungstechnik'32, all dieses nicht kommentierte Darbieten einer selbst noch bei splitternden Fenstern und äußerster Lebensmittelrationierung heilen und offenbar durch nichts auf der Welt zu irritierenden Bürgerwelt auf der einen Seite, und all das für derlei unbeirrbare Käseglocken-Seligkeit nötige Ausblenden jener Geräusche auf der anderen Seite, die von weitermarschierenden Knobelbechern herrührten, von deportierten Kirchenglocken oder von den kaum zu überhörenden Sägegeräuschen am Ast der geschilderten Bürgerlichkeit - dies erklärt sehr viel von dem nur prima vista frappierenden Erfolg 'von rechts bis links'. "Von Lagern und Millionen von Toten, von Verbrennungsöfen und Foltern ist nicht die Rede."33 Von sehr viel mehr noch ist in Kempowskis 'Erinnerungskino'34 nicht die Rede - genau besehen von all jenem nicht, das ohne Kommentar nicht schuldlos hätte erwähnt oder gar beschrieben werden können. Um dies an einem einzigen, überaus symptomatischen Satz zu zeigen, der in Tadellöser $\&$ Wolff zu finden ist und der in diesem ganzen Roman die einzige Allusion an das 'Holocaust' erlaubt, hier freilich nachgerade zynisch umgebogen in die Dimension privater Kriminalität: "in Auschwitz, bei Kattowitz, da habe sich auf der Straße ein blutiges Ehedrama abgespielt."35

Man meine nicht, es handle sich hier um ein mit bösartiger Geduld aufgespürtes und dem angestammten Kontext entwundenes Zitat: dichter nämlich an große oder schreckliche Ereignisse pflegt Kempowski nirgends heranzugehen als äußerstenfalls bis auf eine Straße, die, vielleicht, zu den Vernichtungslagern führte. ${ }^{36}$ - Durch die Form der indirekten Rede wie im zitierten Satz, sonst auch häufig durch die Form der Ellipse (für die jüngeren Leser bedeutet sie eine erläuterungsbedürftige Aposiopese) oder durch 'listige Konjunktive'37 durch solche Formen in der Realitätsverbürgung noch zusätzlich eingeschränkt, verdampft Kempowski all jene Daten, die im Ploetz im Fettdruck stehen, bis zu einer Unkenntlichkeit, die nur noch dem damals wachen und heute erinnerungsbereiten Zeitgenossen die Möglichkeit zur 'Rückblende' läßt. Wer sich indessen nicht erinnern $k a n n$ oder mag, den vermögen jene vielfach verpuppten Spurenelemente über die in vollendeter Diskretion angesprochenen Zusammenhänge nicht aufzuklären; und es besteht auch keine Gefahr, daß ein solcher Leser sich aufgestört fühlen müßte.

Ohne Zweifel: Kempowski hat es zur Virtuosität darin gebracht, es jedem Leser recht $\mathrm{zu}$ machen und es mit keinem zu verderben. Daß er von Lesern aller 
Generationen, aller Schichten, aller Landsmannschaften, aller intellektueller Schattierungen, ja sogar und vor allem jeglicher politischer Couleur geschätzt wird; daß er tatsächlich längst 'Ein Wallraff für die ganze Familie'38 geworden ist - das hängt sehr eng damit zusammen, daß er auf seine Art Vergangenheitsbewältigung betreibt: so nämlich, daß von den Totenlisten und Schlagzeilen geschwiegen und stattdessen von den Reklameverslein der Stollwerck-Schokolade und von Schlagerzeilen wie dem Mann mit dem Koks ${ }^{39}$ gesprochen wird. Der Kontext derartiger 'Realitätsvokabeln' (Hermann Broch), welche 'Kolorit und Atmosphäre von Ort und Zeit' verbürgen sollen, 40 ist von jedem einzelnen Leser herzustellen - wie überhaupt der Leser erst duch selbsttätige Verknüpfung der diskontinuierlich präsentierten Erzählpartikel den Romanzusammenhang und die epische Totalität konstituieren muß; was hier dem einen wegen Erinnerungslücken oder wegen mangelnder Erlebnisparallelen eine taube Nuß bleiben mag, das wird dem anderen möglicherweise zu einer ganzen Flut von Assoziationen, zu 'Aha'-Effekten 41 und zu einem spontanen Mitteilungsbedürfnis auf der Ebene des 'Weißt Du noch?' verhelfen. Für beide Lesertypen und ebenso für die zwischen diesen Extremen anzusiedelnden Leser gilt die Feststellung von Uwe Herms, Walter Kempowski sei der Schriftsteller der Siebziger-Jahre, "weil er dem sich immer mehr durchsetzenden antireformerisch eingestellten Bürgertum hilft, seine Tradition über sein gedrucktes Sprachbild wiederzufinden." 42

Die Frage bleibt nur, ob sich in Kempowskis beziehungsreichem Magazinieren genau recherchierter Reminiszenzen, ob sich in der mit der 'Monomanie eines inspirierten Bürokraten'43 aufgehäuften Ansammlung von historischem Treibgut tatsächlich die Tradition deutschen Bürgertums zeige - oder ob sie sich hier nur finden, also ins poröse Erinnerungsangebot projizieren lasse, je und je verschieden, immer aber als ein zur persönlichen Beruhigung und zur biographischen Stilisierung geeignetes mixtum compositum, dessen entscheidende Bestandteile nicht vom Autor stammen, sondern von seinen Lesern selbst. Der Erfolg von Kempowskis Romanen wird somit - in einem ganz spezifischen Sinne - von den Lesern 'gemacht'. Kempowski, der Autor, erfüllt zwar die sehr konkreten Bedürfnissen entspringende, freilich auch längst schon modisch vereinnahmte Nachfrage nach einer gemodelten Vergangenheitsbewältigung, die alles Böse und Bedrückende milde wegblendet oder nur in betulicher Teichoskopie ahnen läßt - seine Leser aber finden ein Angebot, sich nämlich sogar mit jenen Phasen der 'verlorenen Zeit' wieder versöhnend anzufreunden, die sie beschämender in Erinnerung hatten. Die wacker, oft auch 'krampfig-witzig'44 und 'penetrant humorig'45, ihre Schnacks produzierende Familie Kempowski wird auf diese Weise für den Leser zum Vehikel des eigenen Zurechtkommens mit 'großen' und 'goldigen' Zeiten; und die Leser greifen dankbar nach dem weiten Mantel des Vergessens, Verschweigens und Nichts-gewußt-Habens, der in Kempowskis Romanzyklus alles gütig deckt, was den privaten Daseinskämpfern draußen vor der Tür blieb.

Drinnen, in der überschaubaren Parzelle, wo die Mütter herrschen und die 
Väter fehlen oder nichts zu sagen haben, drinnen, zwischen Vertiko und Lehnstuhl, Schmalztöpfen aus Dänemark und guten deutschen Salamander-Schuhen, bleibt die Kempowski-Welt intakt; mag der Zyklon noch so toben, sein Auge bleibt windstill und unbehelligt. Hier sind die Nachfahren der bismarcktreuen Buchholzens zuhause, ganz existentiell und samt ihren dreisten Vorurteilen und Fehlsichtigkeiten, mit ihren tatsächlichen Wertvorstellungen und mit ihren abgewirtschafteten Idealismen; hier haben sie sich eingerichtet, einmal besser und einmal schlechter - aber immer so, daß die familiäre Fluchtburg uneinnehmbar bleibt; die Zugbrücke geht nur für 'gut Freund' herunter. - Und der Autor läßt diese Zugbrücke ebenso breit und einladend für all seine Leser fallen; er macht seinen Lesern das Angebot, bei den Kempowskis zu Gast zu sein, sich anzufreunden, sich in ihnen wiederzuerkennen, sich mit ihnen zu identifizieren all das in einer nie aufgeregten oder problembefrachteten Atmosphäre humorigschnurriger Vertraulichkeit, in einem Klima animierten Schulter - und auch Schenkelklopfens, auf einer Plauderebene des 'Alles-nicht-so-schlimm-Gewesen', in einer spontanen Solidargemeinschaft derer, die noch einmal davongekommen.

Unterstützend belegt werden solche Urteile durch die typographische Anordnung von Kempowskis Romanen, durch den raschen Wechsel winziger Absätze 'verschmitzter Abbreviaturen' und 'epischer Schnappschüsse'46 - und vieler weißer Zeilen dazwischen; dieser Erzählstil ermöglicht, ja er fordert das Cofabulieren des Lesers, so unterschiedlich dieses je nach Kenntnissen, Erfahrungen und Temperament auch entwickelt sein mag. Wenn es auch paradox klingt: in einem von Autorkommentaren fast gänzlich freien Werk, das auch ohne einen im Text selbst erkennbaren 'impliziten Leser' auskommt, ist es doch erst der über die breite Zugbrücke eingelassene Leser, der, angeregt durch die 'Mosaiksteinchen'47 und Miniatur-artigen 'Diapositive'48 der 'Bilderbogen'49 und 'Impressionsserien'50, die weißen Leerzeilen $\mathrm{zu}$ füllen und damit seinen Roman lesend $\mathrm{zu}$ schreiben hat. $\mathrm{Ob}$ in diesem auf Synthetisierung angelegten 'Puzzle'51 der einzelne Leser die äußerst seltenen, in den Text gleichsam eingeschmuggelten Ironiesignale 52 und die eher verschämten Distanzierungen von der vorgeführten pausbäckigen und blauäugigen Bürgerlichkeit überhaupt erkennen kann, hängt sicherlich von seinen jeweiligen Voraussetzungen an Erfahrungen ab, vor allem an Erfahrungen mit Literatur; aber der durch selbständige Komplettierung, Anreicherung oder Einvernahme erst vollständig gemachte Lektürevorgang bleibt auch dann 'faszinierend und köstlich', 53 wenn dem Leser nur die Wortwörtlichkeit dieser Romane zugänglich ist, also die detailgespickte und sammelwütig arrangierte Oberfläche einer in fingiertem Realismus geschilderten Welt. 54

Dieser Oberfläche hat der Leser, jeder einzelne Leser auf seine persönliche Art, die mit Bedacht ausgesparte Tiefenstruktur selbst zu verleihen; und die Romantexte Kempowskis erlauben eine Ausmalung der vorgegebenen Rasterpunkte sowohl dem, der dabei milde mit sich selbst umgehen will, wie auch jenem, der bereit ist, die über viele Kleinigkeiten evozierte Erinnerung an Großes 
und Schreckliches auszuhalten. Kempowskis akribisch vorbereiteter Nachhilfeunterricht über die neuen und nicht mehr so neuen Leiden eines alten Standes und über dessen trotzig-gerissen alle Veränderungen draußen überdauernde und drinnen bewahrende Mentalitätsmuster lädt zu jener Art von interpolierendem Lesen ein, die den literarischen Erfolg geradezu unausweichlich macht: käme nämlich der Leser nicht zu dem berühmten Lesevergnügen, es fiele auf ihn selbst zurück, nicht aber auf den Autor.

Kempowskis Romane - und dies hat als ein weiterer Garantiefaktor seines Erfolges sehr eng mit dem eben Gesagten zu tun - kann man wie andere Bücher auch in größeren Abschnitten oder in einem Zuge lesen; man kann aber zudem, und das ist bei wenigen anderen Büchern möglich und sinnvoll, den Lektürevorgang jederzeit und auf jeder beliebigen Seite ohne Verlust an Erzählkontext unterbrechen und anderswo neu aufnehmen. An 'Spannung' geht bei einer solchen vom Druckbild bereits offerierten Portionierung nichts verloren; 'Spannung' ist eine in diesen Romanen wohlüberlegt ausgesparte Kategorie; und ebensowenig ist zu spüren vom 'langen Atem', der den Lehrbuch-Epiker kennzeichnen würde. Menge und Tempo der Kempowski-Lektüre bleiben also dem Einzelleser ebenso freigestellt wie die inhaltliche und auch die ideologische Besetzung. Kempowskis Stil, im einzelnen so wirklichkeitsgesättigt und im groBen Ganzen so realitätenscheu, liefert seinen Lesern Spielmaterial zu Pastiches, die sich vom Original rasch entfernen, ihre eigenen Kontinuitäten entwickeln und auch jene Bereiche umfassen können, die der Autor unkommentiert gelassen oder nur angedeutet oder die er gänzlich ausgespart hat.

Kempowskis auf elliptische Fortsetzung angelegte Manier zu kopieren oder zu parodieren, fiele nicht schwer. Und es gelänge sicher auch, würde nur geduldig genug recherchiert, mit dieser Manier auch frühere Jahrhunderte als das zwanzigste oder das ausgehende neunzehnte vorzuführen. Freilich, es gelänge auch etwa im achtzehnten Jahrhundert nur um den Preis bewußten Verzichts auf die dicken Linien der Landkarten und auf die fetten Zahlen im Ploetz; es gelänge also nur mit jenen Abertausenden von Pünktchen und Kleinst-Radierungen, denen wiederum der Leser Verbindungen zu schaffen und Farbe zu geben hätte - und wiederum fiele dies abhängig vom jeweiligen Kenntnisstand aus; es verlöre freilich an persönlich-familiären Vergleichsbezügen, je weiter eine solche Chronik in die fernere Vergangenheit zurückführte. Der autor absconditus verlangt für seine facta non bruta den lector supplens.

Sieht man einmal von der sicher bald gefüllten Lücke von 1918 bis $1933 \mathrm{ab}$, so hat Kempowskis bisheriger Romanzyklus einen Zeitraum von reichlich sechzig Jahren zum Gegenstand; einen Zeitraum, den auch noch die Jüngeren als einen überschaubar-nahen, aus den Erzählungen der eigenen Familie, aus Geschichtsbüchern oder Filmen gegenwärtig haben (können). Diese historische Nähe bildet eine Bedingung der Möglichkeit 'erfolgreichen' Lesens - und mithin eine Bedingung der Möglichkeit für den Erfolg Kempowskis. Er, der 'Wallraff für die ganze Familie', bietet mit seinem portionierten und porösen Stil ein auf 
individuelle Ergänzung, Vervollständigung, potenzierte Privatisierung und cofabulierende Anverwandlung angelegtes, von vornherein also vielfach gestaffeltes Leseangebot, das 'von rechts bis links', von Jung und Alt, von Urlaubsschmökerern wie von Zeitpuls-Forschern, von Ironie-Sensiblen wie von Oberflächen-Befriedigten, von 'restringierten' wie von 'elaborierten' Lesern, kurz, das von jedermann mit einigem Genuß wahrgenommen und persönlich ausstaffiert werden kann - auch mit erheblichem Lerngewinn an nicht mehr präsenten oder nie registrierten Fakten einer zwar familiär begrenzten, aber datensatten Wirklichkeit. Daß es sich hierbei nicht um die ganze (historische) Wirklichkeit handeln kann und handeln darf, daß hier kein Realismus repräsentativer Totalität vorliegt, daß also der Leser überall einschlüpfen kann und soll, daß er das in minuziösen Situations-Facetten wiedergegebene Familiäre der Kempowskis nocheinmal familiarisieren soll - alles dies läßt den vorgeblichen Verismus Kempowskis sogleich ins mehr oder weniger reine Lesevergnügen überspringen, in eine ganze Skala von Rezeptionsformen also, die begrenzt ist von den Extremen ernsthafter Problemverarbeitung und einem trivialen bis kitschigen Sich-Einlullen.

Der Erfolg der Romane Walter Kempowskis hängt natürlich auch mit dem für eine solche Art privatisierter Vergangenheitsbewältigung günstigen kairós der siebziger Jahre zusammen. Und er hängt damit zusammen, daß hier ein Lesefutter - 'immer was zum Beissen'55 - verabreicht wird, bei dem sich niemand den Magen der Erinnerung verderben, das vielmehr jedermann mit Ingredienzien nach eigenem Gusto zurichten und dann ohne Reue genießen kann. Kempowskis Erfolg ist also in einem hohen Maße dadurch begründet, daß in einer oftmals und wohl auch zurecht als geschichtsvergessen bezeichneten Zeit seine Bücher einen willkommenen Geschichtsbuchersatz aus historischem Kleingeld bieten, der - 'faszinierend und köstlich', unanstößig und 'seltsam zeitlos'56 - nichts Angestrengtes hat und auch vom Leser keine Anstrengung fordert, vor allem nicht die Anstrengung der Trauerarbeit. Davor schützt ihn die von Kempowski selbst als 'mein Thema' bezeichnete 'Atmosphäre der Harmlosigkeit' 57 , in der Leser-Vorurteile eher bestätigt als korrigiert werden, 58 eine Atmosphäre von 'Ordnung', 59 die auch auf den Leser entlastend wirkt. Kempowskis Romanzyklus präsentiert das individuelle Exempel einer angeblich durchschnittlichen Familie - ein Exempel, dessen Paradigmawechsel in private Einvernahme und Anverwandlung jedem neuen Leser mühelos gelingt.

$\mathrm{Da} \beta$ jedes literarische Werk nicht nur einen einzigen Modus des Leseverhaltens anbietet, ist eine Binsenweisheit. Kempowski aber hat es in seinen Romanen fertiggebracht, die Palette des Lektüreverhaltens ins prinzipiell Unbegrenzte aufzufächern.

Literarischer Erfolg - um eine weitere Binsenweisheit zu verwenden - ist immer eine Sache auch der Leser und des historischen Klimas. Kempowski hat es verstanden, seine Leser in einem Maß supplierend und cofabulierend am Prozeß lesender Herstellung von Wirklichkeit zu beteiligen wie kein anderer 
Autor vor ihm. Und er hat dabei das Klima der Zeit genützt, welche Geschichte leichter erträgt, wird sie in Geschichtchen serviert.

Literarische Erfolge - eine dritte Binsenweisheit - kann es auch gegen die Leser oder den literarischen Geschmack oder die herrschende Meinung geben; Grass' Blechtrommel wäre ein Beispiel dafür. Kempowskis Erfolg ist gewiß nicht auf diese Weise entstanden.

Sein Erfolg rückt ihn deshalb noch nicht ohne weiteres in die Nähe Simmels; aber die Klasse von Grass bleibt ihm sicherlich - in doppeltem Sinne ebenso versperrt.

Kempowski hat eine Serie von Erfolgs-Romanen geschrieben. Der Erfolg wird ihm seriell treu bleiben.

\section{Anmerkungen}

1 Leicht veränderter Habilitationsvortrag (18.7.1979). Die Untersuchung stützt sich auf vergleichsweise enges, jedoch repräsentatives Textmaterial. Seither erschienene Texte, eine weitere Verfilmung (Ein Kapitel für sich) und andere literarische Aktivitäten Kempowskis blieben unberücksichtigt; an den hier vorgetragenen Thesen hätten sie nichts verändert.

2 Diesen Terminus gebraucht Walter Kempowski im "Gespräch mit dem Autor", das Dieter E. Zimmer führte - in Die Zeit, 5. April 1974. Tags zuvor, am 4. April 1974, äußerte W.K. in einem Interview der Welt mit Hilke Prillmann auf deren Frage, ob die Antwortensammlungen als 'literarisches Abfallprodukt' zu werten seien: 'Nein; vielmehr sind es Abstecher, die direkt mit meinen Romanen verbunden sind. Ich bearbeite hier Themen, die ich in dieser Weise in den Romanen nicht behandeln konnte oder wollte."

3 Nach: Michael Berger, "Noch einmal davongekommen. Walter Kempowskis Nachkriegszeit-Report", Kölner Stadtanzeiger, 28. September 1972.

4 W. K. im Gespräch mit Claudio Isani. In Der Abend, 18. September 1975. (Titel: "Mein Gott, Walter! Als die Diskussion zu Ende war: Kempowski - ein Kapitel für sich".)

5 Walter Jens ("Momos"), "Von Folter und Verbrennung keine Rede", Die Zeit, 9. Mai 1975.

6. Das Zeit-magazin hatte W. K. bereits am 29. September 1972 in einem Artikel von Jost Nolte vorgestellt: "Ein Liberaler: Walter Kempowski".

7 Nach: Margarete von Schwarzkopf, "Der liberale Einzelgänger mit den zwei Berufungen. Die WELT sprach mit Walter Kempowski, Autor der größten deutschen Familien-Chronik und Lehrer in Niedersachsen", Die Welt, 12. Dezember 1976.

8 Uwe Herms, "Ein Wallraff für die ganze Familie. Zu Walter Kempowskis 
neuem, etwas irritierendem Roman", Stuttgarter Zeitung, 9. Oktober 1975.

9 Walter-Scuka-Preis, Niedersächsischer Staatspreis für Literatur, Raabe-Preis, Lessing-Förderpreis.

10 Jost Nolte (= Anm. 6).

11 Peter Wapnewski, "Simplicissimus in Rostock. Ein Buch zum Wiedererkennen. Walter Kempowskis dritter Roman Uns geht's ja noch gold", Die Zeit, 29. September 1972.

12 Helmut de Voss, “ ... und noch einmal Familie Kempowski. Aus großer Zeit", Unser Mecklenburg, Heimatblatt für Mecklenburger und Vorpommern, Juli/August 1978: "All das ist ein bißchen Buddenbrooks in Jeans und mit Rollkragenpulli." W. K. sieht sich selbst - im Gespräch mit Dieter E. Zimmer (= Anm. 2) - "als genauen Kenner von Thomas Manns Buddenbrooks".

13 Hans Joachim Schyle, "Von der Idylle zur Kanonade. Kempowski erzählt seine Familiensage. Ein Zeitmosaik mit Anekdoten und Zitaten", Kölner Stadt-Anzeiger, 14. Oktober 1978: "Kempowski versteht zu erzählen, ein wahrer Flickerlteppich-Fontane".

14 Hans Magnus Enzensberger, "Ein herzloser Schriftsteller". (Ưber Alexander Kluge: Neue Geschichten), Der Spiegel Nr. 1/1978, S. 83: "Darin, $\mathrm{da} ß$ er in die trivialsten Alltagssprachen schlüpft, um so der historischen Erfahrungen habhaft zu werden, ist Alexander Kluge mit Walter Kempowski zu vergleichen, der in seinen Romanen etwas Ähnliches versucht hat. Beide arbeiten sozusagen mit der Pinzette. Aber damit hört ihre Gemeinsamkeit auch schon wieder auf. Denn der eine ist Sammler, der andere Forscher; der eine bringt Verklärung zuwege, der andere Analyse. Kempowski privatisiert die Geschichte, Kluge historisiert die Privatsphäre; was jenem sein Kunstobjekt, ist diesem sein Lernprozeß.

15 Rolf Becker, "Immer unterhaltlich", (über W. K., Aus großer Zeit).Der Spiegel, 18. September 1978.

16 Klaus Wagner, "Die Tadellosen und die Wölfe. Krieg und Kriegsende: Fechners Kempowski-Verfilmung im Fernsehen", Frankfurter Allgemeine Zeitung, 30. April 1975.

17 Wolfgang Preisendanz, "Zum Vorrang des Komischen bei der Darstellung von Geschichtserfahrung in deutschen Romanen unserer Zeit", in Das Komische (Poetik und Hermeneutik Bd. 7). Hrsg. von Wolfgang Preisendanz und Rainer Warning, München 1976, S. 153-164; S. 153.

18 Siegfried Kracauer, zitiert bei: Jürgen Peters, "Kleiner Versuch über den großen Erfolg”. (= Anm. 19), S. 139. 
19 Jürgen Peters, "Kleiner Versuch über den großen Erfolg", in Trivialliteratur (UTB 637). Hrsg. von Annamarie Rucktäschel und Hans Dieter Zimmerman, München 1976, S. 139-168; S. 144.

20 Einen 'Botaniker politischer Zeitgeschichte' nannte W. K. Horst Krüger, "Haben auch Sie Hitler gesehen? Das Kempowski-Prinzip. Nachdenken über eine neue Prosaform", Die Zeit, 13. April 1973.

21 W. K. pflegt "das mosaikartige, pointiert-zugespitzte, dabei doch chronologische Häppchenverfahren" - so: Volker Hage, "Der Mensch ist nicht totzukriegen. Ein Kapitel für sich. Walter Kempowskis neuer Roman", Frankfurter Allgemeine Zeitung, 11. November 1975.

22 Vgl. Herms (=Anm. 8).

23 Vgl. Wagner (= Anm. 16).

24 Kai Niemeyer, "Das kaisertreue Bürgervolk. Walter Kempowski Aus großer Zeit", Abendzeitung (München), 1. September 1978.

25 Krüger (= Anm. 20).

26 Dazu Herms (= Anm. 8): "Die Verfertigung von Büchern ist unaufhaltsam und ihr Inhalt oft unwiderlegbar, der Schriftsteller ist eine Erfassungsstelle, keine moralische Instanz, das Engagement bezieht sich auf Materialbeschaffung und publizierbare Verwertung, Ausgewogenheit im Rahmen der Guckkastenbühne einer kleinbürgerlichen Comédie humaine".

27 W. K. im Gespräch mit Dieter E. Zimmer (= Anm. 2).

28 Ebd.

29 Rolf Becker über Walter Kempowski: "Tadellöser $\mathcal{E}$ Wolff. Herr Hitler müsse es wissen", Der Spiegel, 26. April 1971.

30 Im Gespräch mit Jost Nolte (= Anm. 6) sagte W. K.: "Ich würde mich sehr gerne engagieren. Aber wofür?"

31 Wapnewski (= Anm. 11).

32 Volker Hage, "Walter Kempowskis deutsches Familienalbum. Aus großer Zeit: der Einleitungsband seines Romanzyklus führt in die Zeit nach der Jahrhundertwende", Frankfurter Allgemeine Zeitung, 17. Oktober 1978.

33 Jens (=Anm. 5).

34 K. H. Kramberg, "Aus dem Block. Die Gefängnisse der Kempowskis", Süddeutsche Zeitung, 20. Dezember 1975.

35 W. K., Tadellöser \& Wolff, dtv-Ausgabe, S. 308.

36 Vgl. ebd., S. 203: "Welcher Kommunist hat hier die Büchsen umgeschmissen?" Hinter einem solchen Satz den Kriegsbeginn des Deutschen Reiches 
gegen die Sowjetunion zu erkennen, verlangt vom Leser viel - wohl allzu viel.

37 Becker (= Anm. 15).

38 Herms (= Anm. 8).

39 W. K., Uns geht's ja noch gold, dtv-Ausgabe, S. 326.

40 Alfred Starkmann, "Mit Donnerhall und Wogenprall. Walter Kempowski fügt seiner deutschen Bürgerchronik ein weiteres Kapitel hinzu", Die Welt, 16. September 1978.

41 Georg Ramsegger, "Die Wirklichkeit war schlimmer", National-Zeitung (Basel), 31. März 1976.

42 Herms (= Anm. 8).

43 Ebd.

44 Wapnewski (= Anm. 11).

45 Becker (= Anm. 15).

46 Ebd.

47 Hage (= Anm. 32).

48 Niemeyer (= Anm. 24).

49 Wapnewski (= Anm. 11).

50 Ebd.

51 Vgl. Kramberg (= Anm. 34).

52 Josef Terharn, "Chronik deutschen Bürgertums", Der Tecklenburger (Ibbenbüren), 18. November 1978: "Und mit der feinen Ironie, die inzwischen zu einem Markenzeichen des Autors geworden ist, nennt er das Ganze Aus großer Zeit".

53 Niemeyer (= Anm. 24) - der Albrecht Knaus Verlag verwendete dieses Zitat bezeichnenderweise zur Buchwerbung.

54 Vgl. Hage (= Anm. 32).

55 Martin Gregor-Dellin, "Immer was zum Beissen. Tadellöser \& Wolff von Walter Kempowski: aufgearbeitete Wirklichkeit", Nationalzeitung (Basel), 8. April 1971.

56 Jörg Ulrich, "Im Zeitraffer: Der flotteste Haushalt von ganz Rostock. Der neue Roman von Walter Kempowski: Aus großer Zeit - 1885 bis 1919", Freisinger Tagblatt, 29. September 1978.

57 W.K. im Gespräch mit Claudio Isani (= Anm. 4).

58 Dieser Vorgang ist wiederholt für trivialliterarische Texte nachgewiesen 
worden.

59 W.K. in der Impulse-Sendung, ZDF 31. Juli 1978. 